\title{
Symmetry Breaking in a Plasmonic Metamaterial at Optical Wavelength
}

\author{
André Christ,,,$+ \dagger$ Olivier J. F. Martin, ${ }^{\dagger}$ Yasin Ekinci, ${ }^{\ddagger}$ Nikolai A. Gippius, $, \$, \perp$ \\ and Sergei G. Tikhodeev ${ }^{\perp}$
}

\begin{abstract}
Nanophotonics and Metrology Laboratory, École Polytechnique Fédéral de Lausanne, Station 11, 1015 Lausanne, Switzerland, Laboratory of Metal Physics and Technology, Swiss Federal Institute of Technology Zürich, CH-8093 Zürich, Switzerland, LASMEA, UMR 6602 CNRS, Université Blaise Pascal, 24, av des Landais, 63177 Aubière, France, and A. M. Prokhorov General Physics Institute RAS, Vavilova 38, Moscow 119991, Russia
\end{abstract}

Received February 25, 2008; Revised Manuscript Received May 21, 2008

\begin{abstract}
We numerically study the effect of structural asymmetry in a plasmonic metamaterial made from gold nanowires. It is reported that optically inactive (i.e., optically dark) particle plasmon modes of the symmetric wire lattice are immediately coupled to the radiation field, when a broken structural symmetry is introduced. Such higher order plasmon resonances are characterized by their subradiant nature. They generally reveal long lifetimes and distinct absorption losses. It is shown that the near-field interaction strongly determines these modes.
\end{abstract}

Resonant metallic nanostructures supporting localized surface plasmon polariton modes (i.e., so-called particle plasmons) play a remarkable role in current nanoscience, where their optical properties are the subject of considerable research efforts. ${ }^{1}$ When illuminated at their resonance frequency, extremely strong and confined optical fields can be generated to alter light-matter interactions on the nanoscale. ${ }^{2}$ A very important recent example is the use of periodically arranged metallic wire pairs to mimic so-called magnetic atoms. ${ }^{3-6}$ The observation of magnetic activity generally relies on the fact that wire-wire coupling results in the formation of two energetically separated plasmonic eigenstates with opposite electric dipole moments. The antisymmetric nature of the low-energy mode is thereby essential to provide negative permeability, that is, strong magnetic components which are opposing the external magnetic field. So far, most metamaterial studies have focused on geometries with a symmetrical translation cell as elementary building block, not addressing fundamental effects caused by spatial symmetry breaking. The influence of weakly asymmetric structural elements has been discussed in the case of modulated metal films, ${ }^{7}$ photonic crystal slabs, ${ }^{8}$ and planar metamaterials,${ }^{9}$ however, similar investigations for plasmonic structures are rarely found. A recent example is the investigation of plasmon hybridization in nanoshells with a nonconcentric core. ${ }^{10}$ It was shown that the presence of a core offset, i.e.,

\footnotetext{
* Corresponding author. E-mail: andre.christ@epfl.

† École Polytechique Fédéral de Lausanne.

* Swiss Federal Institute of Technology Zürich.

$\S$ Université Blaise Pascal.

${ }^{\perp}$ A. M. Prokhorov General Physics Institute RAS.
}
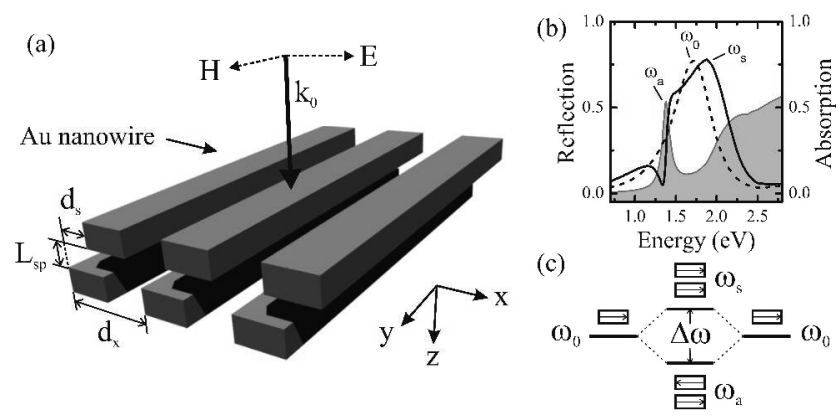

Figure 1. (a) Sketch of the metal nanowire based metamaterial. (b) Reflection (solid line) and absorption spectra (shaded area) for a plasmonic lattice with $L_{\mathrm{sp}}=30 \mathrm{~nm}$ and $d_{\mathrm{s}}=0 \mathrm{~nm}$. The reflection spectrum of a single lattice plane (dotted line) is shown as reference. (c) Illustration of the particle plasmon hybridization scheme.

a structural asymmetry, induces optical activity of higher multipolar plasmons.

In this communication, we numerically analyze the optical response of a plasmonic metamaterial in the presence of structural asymmetry. While the related near-field induced inversion of plasmon hybridization has already been reported, ${ }^{11}$ the present work focuses on the excitation of socalled dark plasmon modes (also known as trapped modes). ${ }^{9}$ It is shown that these spectrally narrow additional modes possess a unique electromagnetic field distribution and only become optically active when a broken spatial symmetry is introduced. The described fundamental phenomena might pave the way for novel metamaterial applications.

As shown in Figure 1, the studied model system consists of two identical nanowire gratings separated by a finite 

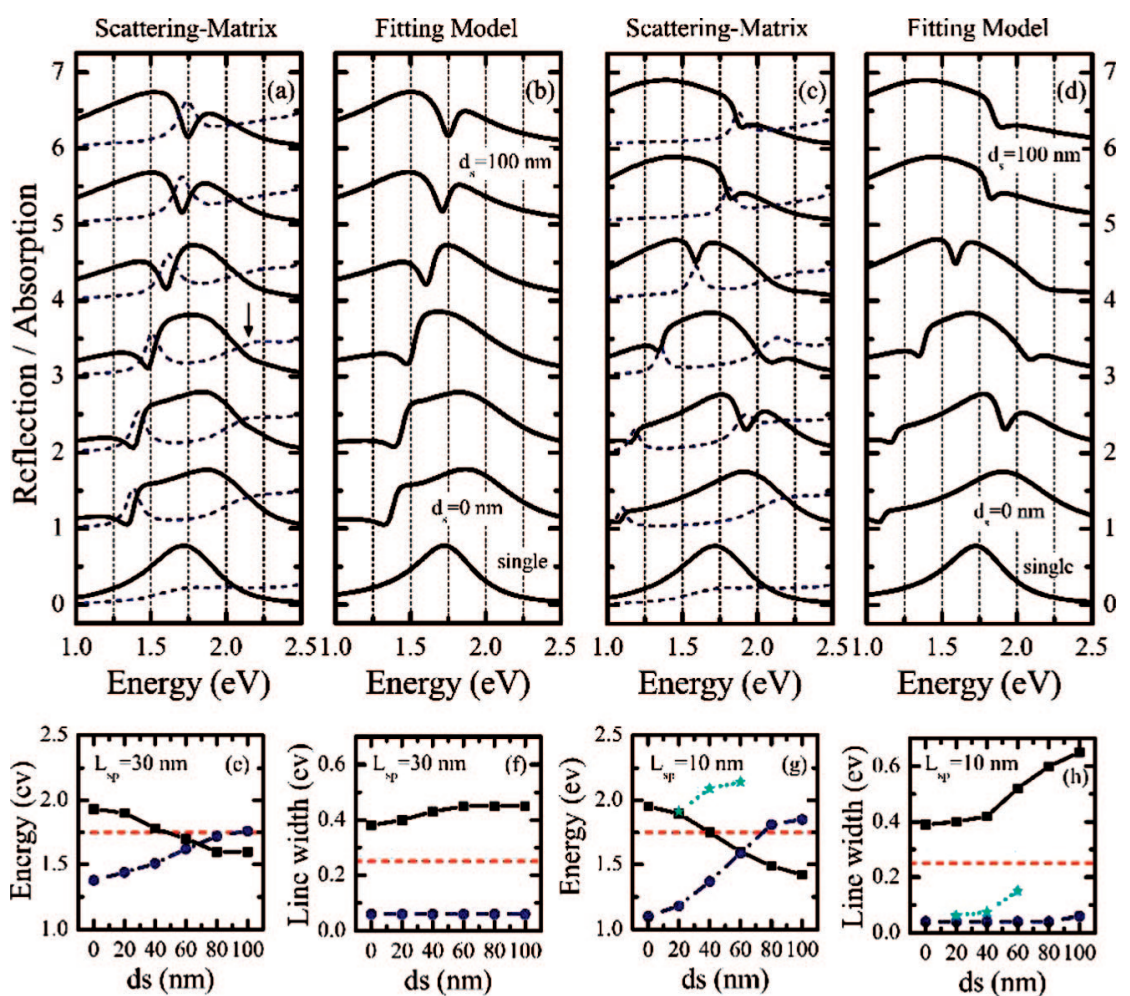

Figure 2. Reflection (solid lines) and absorption spectra (dashed lines) for lattice structures with $L_{\mathrm{sp}}=30 \mathrm{~nm}(\mathrm{a})$ and $L_{\mathrm{sp}}=10 \mathrm{~nm}$ (c) are shown (normal incidence, $p$-polarization). The lateral shift is increased from $d_{\mathrm{s}}=0$ to $100 \mathrm{~nm}$ in steps of $20 \mathrm{~nm}$. The lowest curves display the spectra of a single layer reference. Fitted results can be found in panels b and d. Extracted fit parameters (mode position, line width) for both geometries are plotted in panels $\mathrm{e}-\mathrm{h}$.

distance $L_{\mathrm{sp}}$. The nanowires with a cross section of $100 \times$ $15 \mathrm{~nm}^{2}$ (invariant along the $y$ axis) are embedded into a homogeneous silica matrix to avoid any interface effects. The wire period of $d_{\mathrm{x}}=200 \mathrm{~nm}$ is kept constant, and the incident electromagnetic radiation is assumed normal to the $x-y$ plane. The periods are chosen such that all higher order diffraction channels are evanescent within the considered spectral range. A broken structural symmetry is introduced by displacing one lattice plane by $d_{\mathrm{s}}$ along the $x$ axis. Note that the considered wire based metamaterial can be interpreted in terms of a so-called plasmonic superlattice. ${ }^{12}$ In contrast to a simple lattice with a single wire per translation cell, the superlattice translation cell is now composed of two wires. The supercell period is specified by $d_{x}$, while $d_{\mathrm{s}}$ describes the lateral wire displacement within the individual supercell. Numerical modeling was performed using a scattering-matrix formalism ${ }^{13}$ which is generally well-suited for the study of a periodically structured multilayer medium. Only the geometrical properties and the dielectric permittivities of silica $\left(\varepsilon_{\mathrm{s}}=2.13\right)$ and gold $^{14}$ are used as input parameters. The propagation direction of the incident light field is along the $z$ axis, if not stated otherwise. Only $p$-polarization (magnetic field oriented parallel to the nanowires) is considered, since no plasmonic effects are expected for $s$-polarization.

The formation of collective polaritonic eigenmodes due to coupling of the particle plasmons of the individual lattice planes is highlighted in Figure 1b. The degeneracy of the single layer plasmon is lifted which directly results in a much richer reflection spectrum of the aligned $\left(d_{\mathrm{s}}=0 \mathrm{~nm}\right)$ double layer structure. The near-field induced mode splitting can be understood in terms of plasmon hybridization. ${ }^{15,16}$ As schematically drawn in Figure 1c, the new eigenstates of the coupled structure differ in their symmetry. The antisymmetric plasmon mode is characterized by an out-of-phase oscillation of the free electrons and is therefore lowered in energy. In contrast, the energy of the symmetric mode is increased due to the in-phase oscillation of the electrons. While plasmon hybridization is a near-field induced phenomenon, radiative interaction has to be taken into account when addressing the observed lifetime modifications. The antisymmetric plasmon mode is subradiant in character, that is, the resonance couples weakly to the radiation field and radiative decay is slowed down. Moreover, the spectrally narrow subradiant mode interferes with the radiatively broadened symmetric (superradiant) plasmon mode. Their spectral overlap results in a dispersive, Fano-like line shape modification.

The influence of symmetry breaking is demonstrated in Figure 2 by considering a lateral lattice displacement $\left(d_{\mathrm{s}} \neq\right.$ $0 \mathrm{~nm}$ ) and $L_{\mathrm{sp}}=30 \mathrm{~nm}$. The first striking observation in Figure 2a is that the plasmon hybridization scheme is inverted because of the altered near-field coupling. ${ }^{11}$ In the case of an aligned structure, because the near-field interaction between the stacked wires is repulsive for the symmetric mode and attractive for the antisymmetric mode, the energy of the symmetric mode is higher than that of the antisymmetric one. This fact is changed when the nanowires are laterally displaced with respect to each other. The Coulomb interaction between the wires is modified and, for sufficient 
displacement $d_{\mathrm{s}}$, can be expected to change sign. This causes the antisymmetric mode (dip in the reflection spectrum) to have higher energy than the symmetric plasmon mode. The resonance position and their spectral width can be obtained by fitting the far-field reflection spectra $E(\omega)=|r(\omega)|^{2}$ by a phenomenological model ${ }^{11,17,18}$ assuming

$$
r(\omega)=a_{r}+\sum_{j} \frac{-b_{j} \Gamma_{j} e^{i \varphi_{j}}}{\hbar \omega-E_{j}+i \Gamma_{j}}
$$

where $a_{r}$ specifies the amplitude of a constant background, and $b_{j}$ and $\phi_{j}$ characterize the amplitude and the phase of the two localized surface plasmon eigenmodes. The spectral position and the line width of the individual resonances are specified by $E_{j}$ and $\Gamma_{j}$, respectively. Figure $2 \mathrm{~b}$ displays the corresponding fits of the numerically calculated spectra. While a single pole is sufficient for the fit of the one layer reference lattice, the double layer structure requires a twopole model. The extracted resonance positions and corresponding linewidths are shown in Figure 2e,f to highlight the induced modifications. Although the fit shows a reasonable spectral agreement, small deviations can be found on the high energy tails (above $1.8 \mathrm{eV}$ ) of the reflection spectra in case of structural asymmetry. The numerical results exhibit a weakly pronounced dip which is not found in the model spectra (e.g., arrow in Figure 2b).

To clarify the origin of the observed spectral deviations, reflection and absorption spectra for wire structures with a slightly changed lattice spacing of $L_{\mathrm{sp}}=10 \mathrm{~nm}$ are displayed in Figure 2c. Similar to Figure 2a, the structural asymmetry is again introduced by increasing $d_{\mathrm{s}}$ from 0 to $100 \mathrm{~nm}$. In particular, the enhanced near-field interaction between the wires results in two distinct spectral modifications. First, the formation of symmetric and antisymmetric eigenmodes shows a much larger energy splitting, and as a result, the induced energy shifts are increased when changing $d_{\mathrm{s}}$. Second, the spectra indicate an additional resonance at higher energies. The small dip, which appeared in Figure 2a, is now strongly enhanced. Because of a Fano-type interference with the blue-shifted symmetric eigenmode, the absorptive additional resonance leads to a sharp and pronounced dip in the reflection spectra. Note that the appearing third spectral feature requires the use of a three-pole model to fit the reflection spectra; that is, three modes have to be taken into account in eq 1 . The extracted mode positions and corresponding linewidths are shown in Figure 2d.

The calculated spectral response as a function of $L_{\mathrm{sp}}$ is shown in Figure 3 for structures with a slightly asymmetric translation cell assuming $d_{\mathrm{s}}=20 \mathrm{~nm}$. The displayed spectra reveal that the excitation of the discussed additional resonance critically depends on the strength of the near-field interaction between the stacked wires. In the case of $L_{\mathrm{sp}}=$ $30 \mathrm{~nm}$, only the lowest order plasmon modes (symmetric and antisymmetric) are strongly pronounced. The lateral lattice displacement results in a slight modification of the resonance positions in comparison with the aligned structure. Moreover, a first indication of the additional mode is already found. The mode excitation induces a very weak dip in the reflection spectra at approximately $2.1 \mathrm{eV}$, close to the onset of the interband transitions. A stepwise reduction of the

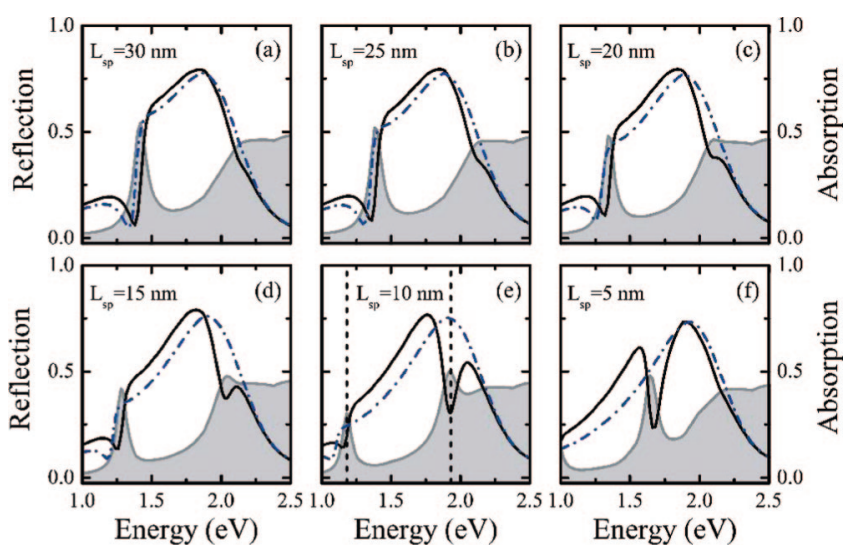

Figure 3. Reflection (solid lines) and absorption spectra (shaded area) for structures with a lateral displacement of $d_{\mathrm{s}}=20 \mathrm{~nm}$. The vertical wire separation is decreased from $L_{\mathrm{sp}}=30$ (a) to $5 \mathrm{~nm}$ (f). The dotted vertical lines in panel e indicate the spectral positions for which the field distributions are shown in Figure 4. Reflection spectra for lattice structures with $d_{\mathrm{s}}=0 \mathrm{~nm}$ are shown as reference (dash-dotted lines).

spacer thickness $L_{\mathrm{sp}}$ (i.e., stronger near-field interaction) however increases the coupling efficiency of the additional mode to the radiation field. The related dip (peak) in the reflection (absorption) spectra becomes more pronounced, while simultaneously shifting to lower energies. The spectral interference of the additional mode with the spectrally broad symmetric plasmon mode finally results in a very narrow feature for structures with $L_{\mathrm{sp}}=5 \mathrm{~nm}$. Now, the resonance position of the additional mode is well-separated from the absorption band starting at $2 \mathrm{eV}$. In a first step, the antisymmetric nature of the additional plasmon mode can already be extracted without plotting the corresponding electric or magnetic field distributions. In particular, the resonance shows a similar behavior as the low energy plasmon mode, which is indeed antisymmetric. Although symmetry breaking leads to the opening of radiative decay channels, the additional resonance is strongly absorptive and is characterized by its narrow spectral line width (i.e., long lifetime). Another indication is the red shift of the resonance when decreasing $L_{\mathrm{sp}}$. The charge distribution along the coupled wires (along the $x$ axis) must be antisymmetric, so that the direct Coulomb interaction weakens the electron restoring forces and reduces the resonance energy for small wire separations.

The physical origin of the observed narrow resonances, that is, their characteristic symmetry properties, can be immediately deduced from the calculated field distributions, as presented in Figure 4. For the displayed contour plots, a nanowire separation of $L_{\mathrm{sp}}=30 \mathrm{~nm}$ and a displacement of $d_{\mathrm{s}}=20 \mathrm{~nm}$ have been chosen. The electric and magnetic field amplitudes are shown for photon energies of 1.18 and $1.93 \mathrm{eV}$ to compare the characteristic properties of the two supported absorptive (i.e., subradiant) resonances. The energies correspond to the spectral positions of the related absorption maxima as indicated by the dashed vertical lines in Figure 3e. Because of the fact that the energy oscillates between the electric and the magnetic fields in case of localized resonances, the snap shots of the magnetic fields 

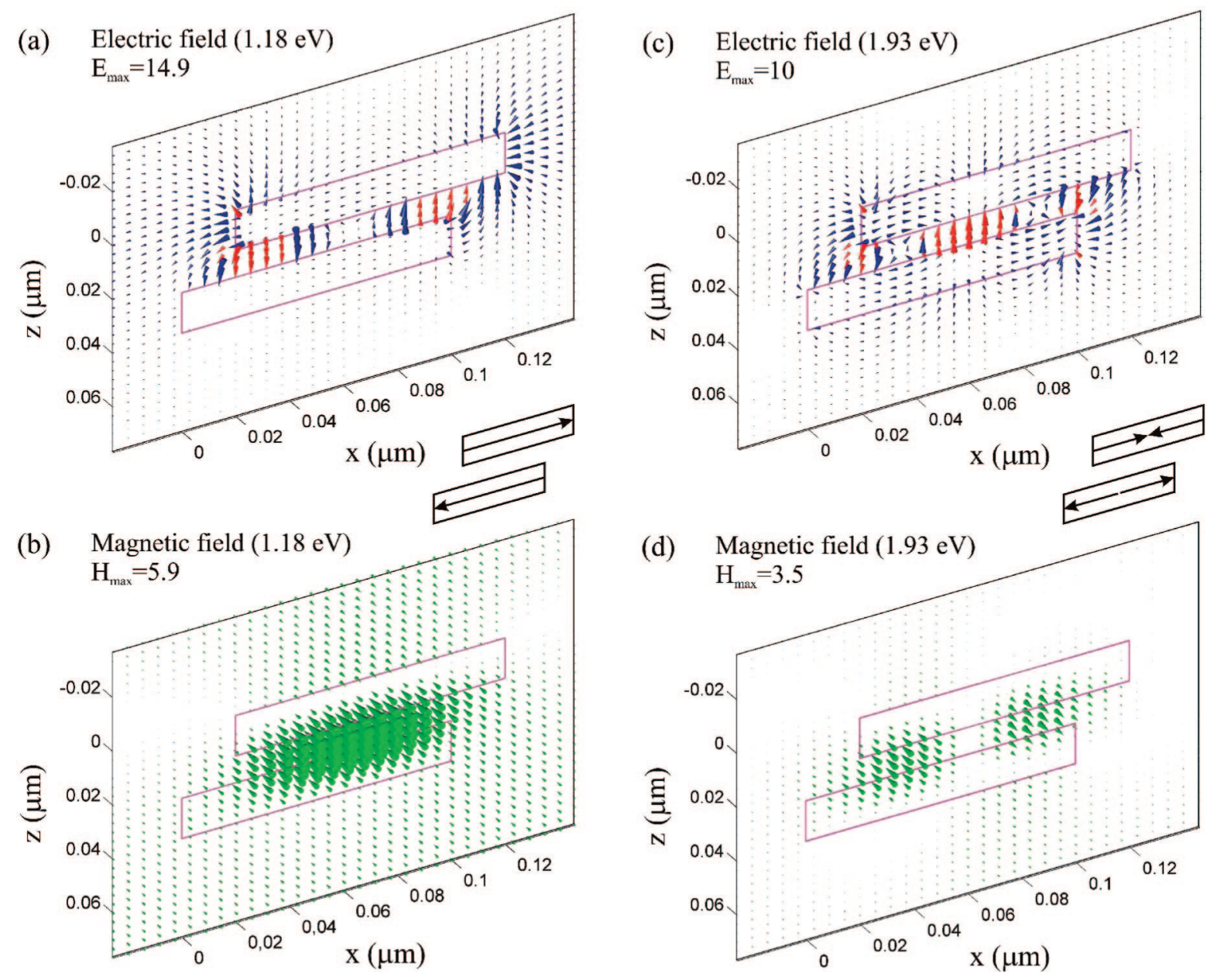

Figure 4. Calculated spatial distribution of the electric and magnetic fields ( $p$-polarization of incoming light at normal incidence). The field amplitudes are shown for photon energies of $1.18 \mathrm{eV}(\mathrm{a}, \mathrm{b})$ and $1.93 \mathrm{eV}(\mathrm{c}, \mathrm{d})$ assuming $L_{\mathrm{sp}}=10 \mathrm{~nm}$. The chosen energies correspond to the resonance positions indicated in panel e of Figure 3. The length of the cones is proportional to the field strength at the central point of each cone, and the field direction is specified by their orientation. The red cones are scaled by a factor of 0.5 . The field distribution is displayed for the moment of time when the intensity (integrated over the displayed area) reaches its maximum. The field magnitudes are normalized to their maximum absolute value measured in units of the incoming field; see values shown in the corresponding panels.

are displayed $T / 4$ later in time than the corresponding electric fields $(\omega=2 \pi / T)$. The low energy resonance at $1.18 \mathrm{eV}$ is clearly antisymmetric in character as shown by the electric field distribution in Figure 4a. The oscillating surface charges and the related displacement currents induced a circular current which directly results in a resonantly enhanced magnetic field in the gap between the adjacent wires. As schematically displayed by the inset, the electric fields within the two wires are opposing each other. Note that the considered plasmonic building blocks can still be used to generate a strong magnetic response and to mimic the socalled magnetic atoms, although the wire geometry already shows a significant structural asymmetry.

The field distribution of the additional resonance at $1.93 \mathrm{eV}$ strongly differs from the lower plasmon mode and shows a much richer spatial structure. Instead of having an electric field minimum in the center of the gap between the displaced wires (Figure 4a), the mode in Figure 4b exhibits a strong electric field enhancement at this position. The characteristic field distribution resembles a snapshot of two current loops oscillating in opposite directions. As clarified by the schematic inset, the electric field indicates the antisymmetric nature of the plasmon mode. In particular, the resonance can be identified as being the second order mode of the so-called magnetic resonance. Instead of a single magnetic field maxima between the wires, the induced circular currents now lead to two maxima in Figure 4d. Note that the magnetic fields at the maxima are phase shifted by $\pi$, as expected for a second order mode. The asymmetric character of the considered collective plasmon mode immediately clarifies why the resonance is optically inactive in perfectly aligned geometries without structural asymmetry: The higher order antisymmetric electron oscillation correlates with the generation of an identical surface charge distribution on each side of the wire pair. Such a charge distribution, indicating the subradiant nature of the mode, cannot be induced by a linear polarized light beam which illuminates the sample at normal incidence.

Up to now, only structural asymmetry has been considered to couple the higher order plasmon mode to the external electromagnetic field. However, an important question to answer is whether oblique illumination will lead to similar 


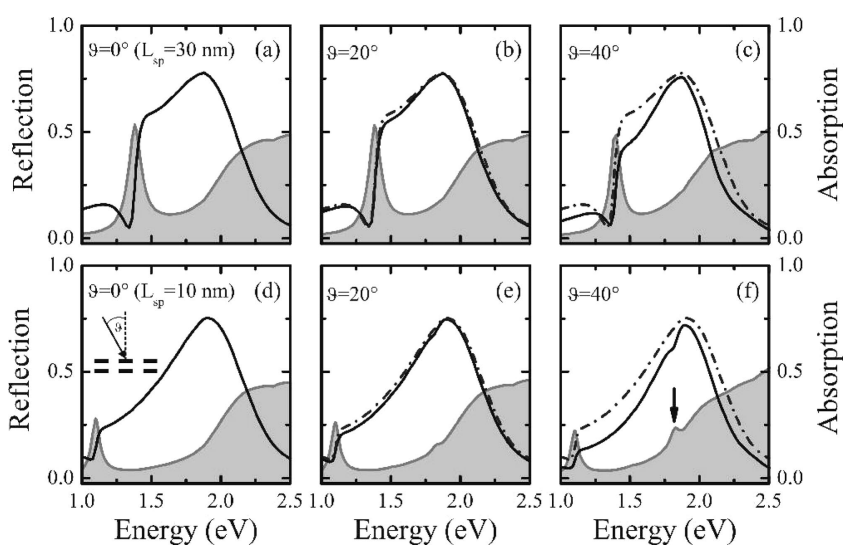

Figure 5. Angular dependent reflection (solid lines) and absorption spectra (shaded area) for structures with $d_{\mathrm{s}}=0 \mathrm{~nm}$. Spectra for spacer thicknesses of $L_{\mathrm{sp}}=30 \mathrm{~nm}(\mathrm{a}-\mathrm{c})$ and $L_{\mathrm{sp}}=10 \mathrm{~nm}(\mathrm{~d}-\mathrm{f})$ are compared. Reflection spectra for normal light incidence are displayed as reference (dash-dotted lines). Inset in d displays the excitation geometry.

results in a perfectly aligned lattice structure with $d_{\mathrm{s}}=0$ $\mathrm{nm}$. For example, it has been shown recently that angular dependent illumination can excite subradiant surface plasmon ${ }^{18}$ or waveguide modes ${ }^{19}$ which are optically inaccessible at normal incidence. That oblique excitation that can also be used to optically address the subradiant higher order plasmon resonance is actually demonstrated in Figure 5. Reflection and absorption spectra for two aligned metamaterial structures $\left(L_{\mathrm{sp}}=30 \mathrm{~nm}\right.$ and $\left.L_{\mathrm{sp}}=10 \mathrm{~nm}\right)$ are compared for $p$-polarization. The incidence angle $\vartheta$ is increased up to $40^{\circ}$. As expected, all plasmonic resonances show a flat dispersion. The resonance positions are not shifted in energy because of their localized nature. The most interesting feature is observed for the small spacer geometry. As indicated by the arrow in Figure $5 \mathrm{f}$ at $1.825 \mathrm{eV}$, the plotted reflection (absorption) spectrum reveals a weakly pronounced dip (peak) nearly in resonance with the spectrally broad symmetric plasmon mode. Although the weak signature of the additional resonance is already visible in Figure 5e, relatively large angles are necessary to achieve a significant coupling to the dark plasmon mode.

In conclusion, we have shown that symmetry breaking in a plasmonic metamaterial can lead to novel material functionalities. In particular, it has been demonstrated that asymmetric double layer lattices allow for the excitation of additional resonances, which are optically inactive in the case of conventional (i.e., symmetric) metamaterials. The additional resonances are identified as being a higher order plasmon mode of antisymmetric origin, that is, with anti- parallel currents along the interacting wire pairs. In addition to the fundamental requirement of an asymmetric translation cell, the opening of radiative decay channels especially relies on the strong near-field coupling between the facing wires. The subradiant additional resonances generally couple very weakly to the external radiation field which leads to spectrally narrow line shapes and extended plasmon lifetimes. Angular dependent calculations reveal that inclined illumination can be used to excite such additional modes even in conventional metamaterials with perfectly aligned wire pairs. The presented plasmonic phenomena are very important from a fundamental point of view: They may provide additional degrees of freedom and have to be considered when designing novel and more complex metamaterials for future applications.

Acknowledgment. This work was financially supported by the European Commission (Grant FP6-202-IST-1507879), the Russian Academy of Sciences, and the Russian Foundation for Basic Research (Grant 06-02-17211).

\section{References}

(1) Kreibig U.; Vollmer, M. Optical Properties of metal Clusters; SpringerVerlag: Berlin, 1995.

(2) Mühlschlegel, P.; Eisler, H. J.; Martin, O. J. F.; Hecht, B.; Pohl, D. W. Science 2005, 308, 1607.

(3) Podolskiy, V.; Sarychev, A.; Shalaev, V. J. Nonlinear Opt. Phys. Mater. 2002, 11, 65.

(4) Dolling, G.; Enkrich, C; Wegener, M.; Zhou, J. F.; Soukoulis, C. M.; Linden, S. Opt. Lett. 2005, 30, 3198.

(5) Zhang, S.; Fan, W.; Malloy, K. J.; Brueck, S. R. J.; Panoiu, N. C.; Osgood, R. M. Opt. Express 2005, 13, 4922.

(6) Linden, S.; Decker, M.; Wegener, M. Phys. Rev. Lett. 2006, 97, 083902.

(7) Gérard, D.; Salomon, L.; de Fornel, F.; Zayats, A. V. Phys. Rev. B 2004, 69, 113405.

(8) Gippius, N. A.; Tikhodeev, S. G.; Ishihara, T. Phys. Rev. B 2005, 72, 045138.

(9) Fedotov, V. A.; Rose, M.; Prosvirnin, S. L.; Papasimakis, N.; Zheludev, N. I. Phys. Rev. Lett. 2007, 99, 147401.

(10) Wu, Y.; Nordlander, P. J. Chem. Phys. 2006, 125, 124708.

(11) Christ, A.; Ekinci, Y.; Solak, H. H.; Gippius, N. A.; Tikhodeev, S. G.; Martin, O. J. F. Phys. Rev. B 2007, 76, 201405.

(12) Zentgraf, T.; Christ, A.; Kuhl, J.; Gippius, N. A.; Tikhodeev, S. G.; Nau, D.; Giessen, H. Phys. Rev. B 2006, 73, 115103.

(13) Tikhodeev, S. G.; Yablonskii, A. L.; Muljarov, E. A.; Gippius, N. A.; Ishihara, T. Phys. Rev. B 2002, 66, 045102.

(14) Johnson, P. B.; Christy, R. W. Phys. Rev. B 1972, 6, 4370.

(15) Nordlander, P.; Oubre, C.; Prodan, E.; Li, K.; Stockman, M. I. Nano. Lett. 2004, 4, 899.

(16) Liu, N.; Guo, H.; Fu, L.; Kaiser, S.; Schweizer, H.; Giessen, H. Adv. Mater. 2007, 19, 3628.

(17) Fan, S.; Joannopoulos, J. D. Phys. Rev. B 2002, 65, 235112.

(18) Ropers, C.; Park, D. J.; Stibenz, G.; Steinmeyer, G.; Kim, J.; Kim, D. S.; Lienau, C. Phys. Rev. Lett. 2005, 94, 113901.

(19) Christ, A.; Zentgraf, T.; Kuhl, J.; Tikhodeev, S. G.; Gippius, N. A.; Giessen, H. Phys. Rev. B 2004, 70, 125113. 